\title{
Chemical complexity and star-formation in merging galaxies
}

\author{
T. A. Davis ${ }^{1}$, A. Heiderman ${ }^{2}$, D. Iono ${ }^{3}$ and the VIXENS team \\ ${ }^{1}$ European Southern Observatory, Karl-Schwarzschild-Str. 2, 85748, Garching, Germany \\ email: tdavis@eso.org \\ ${ }^{2}$ UT-Austin, Dept. of Astronomy, 2515 Speedway, Stop C1400, Austin, TX 78712, USA \\ ${ }^{3}$ NRO, NAOJ Minamimaki, Minamisaku, Nagano, 384-1305, Japan
}

\begin{abstract}
When galaxies merge the resulting conditions are some of the most extreme found anywhere in nature. Large gas flows, shocks and active black holes all can affect the ISM. Nearby merging galaxies with strong starbursts are the only places where we can conduct detailed study of star formation in conditions that mimic those under which the majority of stars in the universe formed. Here we study molecular gas tracers in 8 galaxies selected from the VIRUS-P Investigation of the eXtreme ENvironments of Starbursts (VIXENS) survey. Each galaxy has also been observed using the integral field unit spectrograph VIRUS-P, allowing us to investigate the relation between the chemical state of the gas, star formation and total gas content. Full details can be found in Heiderman et al. (2011). Here we report on new results obtained from IRAM-30m/NRO-45m 3mm line surveys towards 14 positions in these 8 merging galaxies. We detect $\approx 25$ different molecular transitions towards these objects, many which have never been observed in these galaxies before. Our measurements show that the mean fraction of dense gas increases in later-stage mergers (Fig. 1, left), as does the average optical depth of the gas. Molecular diagnostic diagrams (Fig. 1, right) show that molecular regions we probe are, in general, UV photon dominated. Triggered AGN activity, and/or cosmic ray ionisation (from $\mathrm{SNe}$ II in the starburst) are not yet energetically important in determining the state of the gas.
\end{abstract}

Keywords. galaxies: starburst — galaxies: ISM — ISM: molecules — astrochemistry
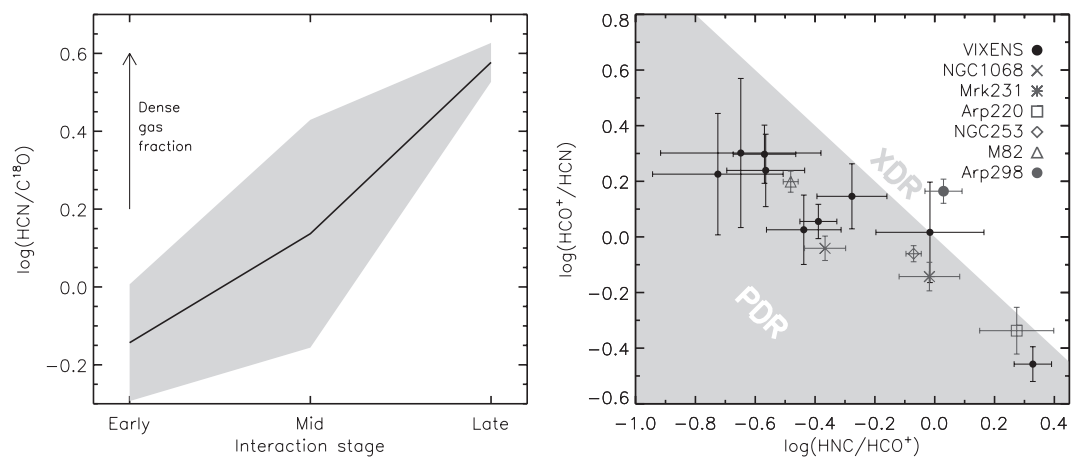

Figure 1. Left: Mean $\mathrm{HCN} / \mathrm{C}^{18} \mathrm{O}$ ratio as a function of interaction stage. Right: Molecular diagnostic diagram after Baan et al. (2008), including the VIXENS galaxies.

\section{References}

Baan et al. 2008, A\&A, 477, 747

Heiderman et al. 2011, in S. Salviander, J. Green, and A. Pawlik (eds.), New Horizons in Astronomy, Proc. Frank N. Bash Symposium 2011. (Online: Proceedings of Science) 